\title{
Le BIM6D comme levier pour une architecture circulaire
}

\author{
Charlotte Dautremont $^{1 *}$, Charlélie Dagnelie ${ }^{2}$, et Sylvie Jancart ${ }^{1}$ \\ ${ }^{1}$ LNA, Faculté d'architecture, Université de Liège, Belgique \\ ${ }^{2}$ Quercus Architecture, Belgique
}

\begin{abstract}
Résumé. Pendant trente ans, l'architecture durable s'est concentrée sur les performances énergétiques des bâtiments et sur les économies d'énergies. Aujourd'hui, d'autres modèles économiques sont étudiés et le modèle linéaire va côtoyer d'ici quelques années le modèle circulaire. Pour des raisons principalement environnementales et économiques, la société retrouve de l'intérêt pour le réemploi et l'économie circulaire. Parallèlement, le processus collaboratif BIM modifie le flux de travail des acteurs de la construction. Cet article s'inscrit dans le cadre d'une recherche sur la relation entre la circularité de (et dans) l'architecture et le processus BIM. Nous voulons à travers lui dégager des pistes pour inclure ce modèle dans la pratique quotidienne des architectes grâce au BIM tout en tenant compte des freins et des leviers liés à ce dernier.
\end{abstract}

Mots-clés. Circularité, BIM6D, Architecture Durable, Réemploi

\begin{abstract}
For thirty years, sustainable architecture has focused on the energy performance of buildings and energy savings. Nowadays, other economic models are being studied and the linear model will stand alongside the circular model in a few years. For mainly environmental and economic reasons, society is once again interested in reuse and circular economy. Simultaneously, the BIM collaborative process is changing the workflow of construction stakeholders, including architects. This article is part of a research on the relationship between the circularity of (and in) architecture and the BIM process. Through it, we want to identify ways to include this model in the daily practice of architects thanks to the BIM, taking into account the brakes and levers linked to the latter.
\end{abstract}

Keywords. Circularity, BIM6D, Sustainable Architecture, Reuse

*cdautremont@uliege.be 


\section{Introduction}

Le processus BIM (Building Information Modeling) révolutionne le flux de travail des acteurs de la construction et particulièrement celui des architectes. Cette lente révolution contribue à de meilleures planification, conception, construction et gestion du projet (Lebegue et Segura, 2015). Malgré ces atouts, le BIM semble être un excellent terrain de promotion des fabricants, avec pour conséquence de transformer les projets d'architecture en catalogue de produits. Ce renouvellement constant va l'encontre du cadre normatif (Directive CE, 2008) sur les déchets et de l'engouement général croissant pour le «zéro déchets ». Si actuellement, nous recyclons beaucoup ( $+/-44 \%$ de déchets municipaux sont recyclés en 2017) (Europa, 2018), nous réutilisons peu ${ }^{1}$. Le BIM, et son lien direct et constant avec les plateformes de produits industriels, ne ferme-t-il pas la porte à une architecture circulaire?

Conscients de la complexité de cette question, nécessitant une étude approfondie, nous allons tenter dans cet article de construire des ponts entre ces des deux domaines, BIM et circularité. Ces ponts seront jalonnés par des freins et des leviers liés à la pratique quotidienne des architectes.

\section{Le contexte d'une double évolution : durabilité et BIM}

Suite au choc pétrolier de 1979 et à la constatation des limites des ressources fossiles, la notion de développement durable apparait dans le rapport de Brundtland ${ }^{2}$ en 1987 avec pour objectif de proposer un développement compatible avec les besoins des futures générations en pérennisant l'utilisation des ressources. Depuis les années 90, l'architecture durable était synonyme d'économie d'énergie et de performance énergétique (PEB) résultant de la Directive CE de 2002.

Malgré cet engouement pour la PEB, d'autres labels sont créés parallèlement, avec pour objectif d'évaluer la durabilité des bâtiments au sens large : LEED (USA), HQE (FR), BREEAM (UK) et à travers eux la notion de l'Analyse du Cycle de Vie (ACV).

Outrepassant la technicité mise en œuvre pour réduire l'impact énergétique, l'ACV considère l'impact environnemental pendant la construction mais aussi pendant l'exploitation et la déconstruction du bâtiment.

L'enjeu de l'architecture durable est désormais le cycle de vie de la construction et de ses composants : avec en amont la question des ressources (disponibilités, provenances et épuisements) et en aval la destination en fin d'exploitation et la notion de déchets. Le nouveau modèle a comme objectifs de maintenir leur valeur initiale et d'éviter de donner un statut de déchets aux éléments et composants, dépassant la construction stricto-sensu. Selon le guide pratique du réemploi (2013), le secteur européen de la construction représente, à lui seul, près de $50 \%$ de la consommation des ressources naturelles et près de $40 \%$ de la production des déchets sur l'ensemble du territoire européen. Avec un tel impact environnemental, des démarches sont mises en place pour aider le secteur vers une transition circulaire. A titre d'exemple, cette transition fait l'objet d'une étude approfondie dans le cadre du projet européen BAMB2020 avec pour objectifs d'amorcer la circularité d'une part par trois changements systémiques majeurs (dans la vision de la conception, dans la définition de valeur et dans la collaboration des acteurs) (BAMB, 2018), mais aussi

\footnotetext{
${ }^{1}$ Dans un communiqué de presse (24/01/2017), les eurodéputés de la commission de l'environnement ont confirmé les nouveaux objectifs pour 2030 avec un pourcentage de recyclage des déchets de $70 \%$.

2 Aussi appelé “Our common future”, rapport rédigé en 1987 par la Commission mondiale sur l'environnement et le développement de l'Organisation des Nations unies.
} 
dans la création de nouveaux outils comme le Passeport des Matériaux (MP) ; outil contenant des informations utiles pour un réemploi futur.

Avec le même objectif commun d'une réduction des déchets, il existe d'autres modèles comme le cas du Lean Management, issu du secteur automobile. Ce modèle a été repris dans le secteur de la construction, plus particulièrement à la phase chantier. Initalement pensé dans le but d'une économie financière, il limite le gaspillage par une meilleure coordination (Arayici et al., 2011).

Parallèlement à ce développement de l'architecture durable, les outils de l'architecte ont eux aussi évolué. Depuis le premier «Personal Computer» en 1981, l'utilisation de l'ordinateur est, dès les années 90, devenue une pratique courante dans les agences d'architecture : en 30 ans, les tables à dessin ont été remplacées par les ordinateurs (Picon, 2016). Cette numérisation du processus entraine une profonde modification du flux du travail : la possibilité de modifier permet une plus grande souplesse dans la conception.

Au cours des années 2000, la productivité s'intensifie grâce à la généralisation de la tridimensionnalité numérique du projet, atteignant son paroxysme avec le BIM. Adoptés dès 2007 par la Norvège, la Finlande et le Danemark, ce n'est qu'en 2014 que la Directive européenne (2014, Article 52) définit les Règles applicables aux communications pour la passation des marchés publics.

L'effervescence exponentielle autour du BIM depuis quelques années traduit les aspirations du secteur de la construction à une réponse systémique de leur profession : le travail collaboratif autour d'un avatar numérique informé.

Bien qu'à l'aube de l'usage de la maquette BIM comme modèle informé et collaboratif (BIM3D), l'acronyme acquière déjà une nouvelle signification : le management ou gestion du bâtiment (BIM4D). Cette nouvelle traduction du BIM amène les notions de temps et de cycle de vie où l'intervention de l'architecte est partie prenante.

Cette double évolution, BIM et durabilité, s'est traduite par des solutions informatiques complémentaires mais séparées. Par conséquent, atteignant un niveau de maturité suffisant des deux parties, nous pensons que le secteur de la construction se trouve à une période propice au changement, tirant profit des retours entre analyse, monitoring et observations, tel qu'illustré dans la Figure 1 (Delmée et al., 2016) ci-après.

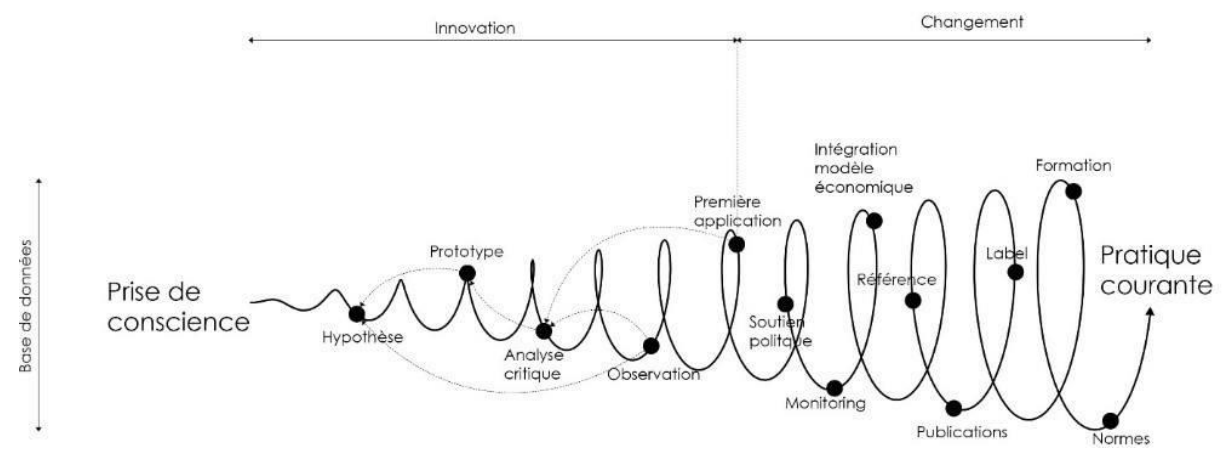

Figure 1. Processus d'implémentation de nouvelles techniques constructives (Delmée et al., 2016).

Cette double évolution n'est-elle pas l'occasion de les réunir au sein du BIM6D ? Rappelons ici que la 4D fait référence à la gestion du temps et au planning tandis que la 5D fait référence à la gestion du coût. Le BIM6D comprend quant à lui tout ce qui est lié au développement durable mais sa définition exacte reste encore incomplète.

Les sections suivantes vont tenter de construire des ponts entre ces deux enjeux contemporains de l'architecture par la mise en lumière de freins et leviers. 


\section{La rencontre : « BIM6D orienté circulaire » ou « circularité numériquement gérée »}

Ces dernières années, l'économie circulaire est devenue le cheval de bataille des politiques nationales et européennes. Le secteur de construction y joue un rôle central dû à son impact sur les ressources et les déchets. Pourtant aujourd'hui, les outils d'intégration du réemploi dans le processus de conception architecturale manquent à l'appel. Ils sont soit inexistants, anecdotiques, partiels ou en cours de développement (MP par BAMB). De plus, en Belgique, nous pouvons souligner la situation révélatrice du statut marginal et/ou pilote de ces projets comme le projet de l'Athénée Riva Bella de Braine-l'Alleud, par l'atelier Alain Richard, prise comme référence.

Gobbo constate : Cette considération de déchets ou de ressources futurs que nous mettons en ouvre aujourd'hui est rarement prise en compte par les bureaux d'architecture mais également par les autres acteurs du secteur (...) (Gobbo, 2015, p.128) mais également : L'une des difficultés et sources de production de déchets réside dans la définition $d u$ rôle de chaque intervenant mais aussi dans le manque de communication claire entre eux (manque de vocabulaire commun, incompréhension, etc.). Dans ce contexte, il est nécessaire que l'architecte au centre du processus du projet constitue un bon relais d'information entre les divers acteurs et qu'il soit le premier acteur d'une démarche de communication, la première étant la transmission d'informations correctes et complètes (plans, détails, métrés) (Gobbo, 2015, p.104).

La réussite d'une stratégie d'architecture circulaire dépendrait de deux facteurs majeurs :

- une communication avec un échange de données clair et ordonné

- et l'anticipation en amont du projet par l'architecte.

Très vite, grâce à l'interopérabilité et à l'échange d'informations, le BIM peut répondre au premier facteur. En effet, l'architecte est un acteur primordial pour conduire un projet vers une transition circulaire. Aussi, bien que porte d'entrée au réemploi, sa collaboration avec une équipe interdisciplinaire de qualité sera garante de succès, et ce tant pour les projets circulaires que BIM. Le projet d'implémentation du BIM dans l'agence d'architecture britannique John McCall Architects, Liverpool (UK) par Arayici et ses collègues (2011) illustre parfaitement cette richesse de collaboration. Selon nous, de part sa place primordiale dans chacun des domaines, le travail collaboratif est l'un des ponts permettant une rencontre entre l'architecture durable et le BIM.

Avant de s'attarder sur ce levier, nous allons mettre en parallèle le processus BIM et l'architecture durable circulaire. Pour commencer, nous allons préciser le concept de circularité qui aborde deux dimensions :

- «passé »; le réemploi d'éléments ${ }^{3}$ passés pour/dans des projets actuels,

- et « future »; le réemploi d'éléments d'aujourd'hui.

Si nous envisageons le BIM comme outil d'intégration du réemploi dans l'architecture, il est particulièrement adapté pour un réemploi futur (MP du BAMB) mais nuancé pour un réemploi passé puisque pour réemployer des éléments passés dans un projet actuel, l'architecte est confronté à plusieurs freins.

Tout d'abord, le relevé de la situation existante devra dans un projet circulaire prendre un caractère d'expertise, nommé inventaire de la matière. Si cet inventaire n'est pas révélateur d'éléments à réemployer in situ, les acteurs devront réaliser une recherche hors site. Energivore en temps (Delmée, 2017), cet inventaire révèle le second frein qu'est le

\footnotetext{
${ }^{3}$ Nous entendons ici par éléments tout ce qui compose un projet d'architecture de l'espace créé, un composant, un système, etc.
} 
manque de visibilité des éléments à réemployer. La partie 5 évoquera le BIM comme levier à celui-ci. Ensuite, la difficulté de modéliser des éléments non-standards relevés est un obstacle majeur, nécessitant une certaine acquisition par les architectes d'outils paramétriques ou de design algorithmique.

Enfin, cette numérisation demande une information 'non graphique' toute particulière et sous forme de multiples supports afin d'assurer une parfaite compréhension de l'élément de réemploi.

En vue de capitaliser ces informations, un travail collaboratif autour de l'architecte est primordial (projet SpatioData, Ben Rajeb et al., 2014). Selon nous, quatre acteurs ont un rôle majeur dans cette capitalisation : le maître d'ouvrage pour des données empiriques, l'entrepreneur par sa connaissance de terrain, le géomètre pour un relevé de données et enfin les fabricants pour les données techniques telles que l'ACV ou le MP. Malgré cet apport riche en informations, nous craignons que ces analyses demandées aux fabricants, mettent en valeur et soient réservées à des industries d'une certaine importance dû à leur coût (Lame \& Leroy, 2015) et avec pour conséquence une mise en marge de petits fabricants novateurs.

Aussi, avec l'émergence de ces deux concepts de nouveaux profils d'acteurs et d'experts sont envisagés tels que «BIM6D Manager orienté circulaire » ou « Responsable déchets BIM ».

Ainsi, le BIM apparaît comme une excellente porte d'entrée pour amener les architectes et les autres acteurs de la construction au circulaire, sans bouleverser (à nouveau) leur pratique.

\section{Le frein de l'un, meilleur levier pour l'autre ?}

Nonobstant ces constats, nous mettons en parallèle deux listes de freins répartis en 5 familles (technique, juridique, économique, méthodologique et culturelle). Cette liste, présentée dans la Table 1, a été réalisée en nous basant sur "la synthèse exhaustive des freins vis-à-vis du réemploi » de Delmée (2017) et des "Limites et freins associés à l'usage de la maquette numérique » de Marin \& Segura (2014).

Au regard de ces freins, et conscients de la nécessité d'une évolution technique du BIM (cf. IFC), nous allons dans le tableau 2 proposer le BIM6D comme levier à l'architecture circulaire.

\section{Le trio gagnant : création + éthique + collaboration}

Jusqu'à présent, le BIM est encore réservé aux professionnels de la construction alors que le réemploi en construction est plutôt une démarche que font les particuliers. Grâce au BIM6D, l'architecture circulaire quitterait son statut actuel de 'marginal' pour devenir un secteur innovant porté par les experts de la construction. D'autre part, le BIM6D permettra une émulation autour de l'architecture circulaire grâce au cadre légal et aux encouragements des autorités de travailler en BIM pour des projets d'importances en marchés publics. Ce type de marché est souvent repris comme référence à suivre pour les plus petits projets, avec pour conséquence un changement des mentalités par percolation, et ce tant dans le secteur des professionnels qu'auprès des particuliers. 
Tableau 1. Mise en parallèle des freins, limites du réemploi et du BIM basés sur les synthèses de Delmée (2017) et Marin \& Segura (2014).

\section{Freins vis-à-vis du réemploi}

Freins et limites à l'usage du BIM

\section{Techniques \& technologiques}

- $\quad$ peu de filières existantes $\quad$ - $\quad$ manque de maturité de l'IFC =

logistique compliquée

réemploi futur presqu'impossible

avec construction actuelle

perte des informations et

attributs

- malgré une 3D enrichie, mauvaise gestion documentaire

\section{Règlementaires, normatifs \& juridiques (Belgique et France)}

- manque de label $\quad$ - manque de valeur juridique

- $\quad$ flou autour de la responsabilité des acteurs

absence de prescriptions définie de maquette numérique

- manque de traçabilité des matériaux

manque de solutions constructives flou autour de la propriété intellectuelle (transfert de données confidentielles dans un

manque
validées

\section{Économiques}

peu/pas d'offres industrialisées $=$ prix élevé

coût de la main d'œuvre

(spécialisée ou non) élevé

flux de travail classique)

\section{Méthodologiques}

manque de visibilité

offre unicité de matériaux, maigre

panel

nécessite de nouvelles mises en

œuvre (peu documentées)

investissement matériel pour un

parfait échange, même

plateforme

- investissement humain pour passage au BIM

\begin{tabular}{|c|c|}
\hline œuvre (peu documentées) & $\begin{array}{l}\text { - Besoin de définir les usages } \\
\text { futurs de la maquette numérique } \\
=\text { sortir de sa vision singulière } \\
\text { d'acteur }\end{array}$ \\
\hline \multicolumn{2}{|c|}{ Affectifs, institutionnels \& culturels } \\
\hline $\begin{array}{ll}- & \text { manque de confiance du grand } \\
\text { public } \\
\text { - } & \text { barrière « esthétique » } \\
\text { - } & \text { frein psychologique = construire } \\
\text { avec des déchets } \\
\text { - } \\
\text { manque d'enseignements, de } \\
\text { retour, de formation }\end{array}$ & $\begin{array}{ll}\text { - } & \text { gros investissement initial pour } \\
\text { un retour qu'en phase finale } \\
\text { - } \quad \text { rupture entre le rôle de } \\
\text { l'architecte avec l'exploitation } \\
\text { de la maquette numérique à } \\
\text { venir } \\
\text { manque d'élasticité des } \\
\text { habitudes }\end{array}$ \\
\hline
\end{tabular}


Tableau 2. Réflexions sur l'apport du BIM6D comme levier aux freins vis-à-vis du réemploi et du BIM

\begin{tabular}{|c|c|}
\hline Pour le réemploi & Pour le BIM \\
\hline \multicolumn{2}{|l|}{ Techniques et technologiques } \\
\hline $\begin{array}{l}\text { - La « connexion » avec des } \\
\text { plateformes de réemploi (ex. Opalis) } \\
\text { permettrait une meilleure logistique et } \\
\text { le développement de ces initiatives à } \\
\text { plus grande échelle }(+)\end{array}$ & 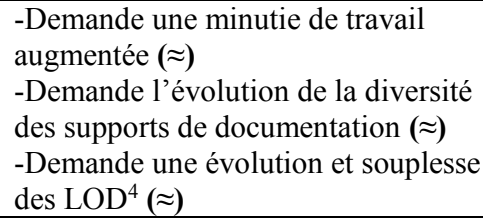 \\
\hline \multicolumn{2}{|c|}{ Règlementaires, normatifs \& juridiques (Belgique et France) } \\
\hline $\begin{array}{l}\text { - Le BIM6D demande de fixer le cadre } \\
\text { réglementaire (normes, labels, } \\
\text { responsabilités, propriété intellectuelle, } \\
\text { etc.) }(\approx) \\
\text { - Il apporte une traçabilité aux } \\
\text { matériaux pour un réemploi futur }(+)\end{array}$ & $\begin{array}{l}\text { - Le BIM6D demande de fixer le } \\
\text { cadre réglementaire (normes, labels, } \\
\text { responsabilités, propriété } \\
\text { intellectuelle, etc.) }(\approx)\end{array}$ \\
\hline \multicolumn{2}{|l|}{ Économiques } \\
\hline $\begin{array}{l}\text { - Le travail collaboratif et le réseau } \\
\text { augmente l'offre/visibilité (principe } \\
\text { offre /demande) }(+)\end{array}$ & $\begin{array}{l}\text { - Le travail collaboratif estompe } \\
\text { l'investissement humain élevé }(+)^{5}\end{array}$ \\
\hline \multicolumn{2}{|l|}{ Méthodologiques } \\
\hline - Meilleure visibilité des offres (+) & $\begin{array}{l}\text { - La durabilité et la circularité } \\
\text { s'appliquent à tous les projets } \\
\text { d'architecture, pas seulement aux } \\
\text { projets d'importance à l'inverse du } \\
\text { BIM (cf. croyance que le BIM est } \\
\text { réservé au gros projets) (+) }\end{array}$ \\
\hline \multicolumn{2}{|l|}{ Affectifs, institutionnels \& culturels } \\
\hline $\begin{array}{l}\text { - BIM6D apporte une « expertise » au } \\
\text { réemploi, permettant de sortir de la } \\
\text { vision «bricolage » }(+) \\
\text { - Il apporte de nouveau challenge à la } \\
\text { création des architectes (par les outils } \\
\text { paramétriques par exemple) }(+) \\
\text { - Se servir de l'engouement du BIM } \\
\text { comme porte d'entrée pour former au } \\
\text { réemploi }(+)\end{array}$ & $\begin{array}{l}\text { - Profitant de l'engouement pour } \\
\text { l'écoconception, le BIM6D devient } \\
\text { un outil à la création (phase initiale } \\
\text { du projet) }(+) \\
\text { - Le réemploi et l'écoconception } \\
\text { sortent l'architecte de la dimension } \\
\text { technico-technologique (créatif) }(+) \\
\text { - Tout changement de paradigme } \\
\text { prend du temps. }(\approx)\end{array}$ \\
\hline $\begin{array}{l}(+) \\
(\approx)\end{array}$ & freins \\
\hline
\end{tabular}

Enfin, le BIM6D donnera une valeur éthique au BIM en l'élevant plus haut qu'un simple moyen technologique ${ }^{6}$ de concevoir.

\footnotetext{
${ }^{4}$ LOD, Level of development. Moyen de mesure du niveau de géométrie d'un objet avec les informations qui lui sont associées.

5 «Constats récurrents lors de formation » où les bureaux voulant passer au BIM nomment 2-3 membres « responsables » pour ne pas geler toute l'activité.
} 
En dehors de toute considération normative, la part créatrice du métier reste souvent l'ultime motivation de l'architecte face aux difficultés grandissantes de son métier. Grâce à l'ouverture du BIM (OPENBIM par BuildingSMART ${ }^{7}$ ), de nouveaux outils de création alliant conception, paramétrisation, construction et informations s'offrent aux architectes. Ces connexions, telles que la connexion Archicad - Rhinoceros3D - Grasshopper, apportent une souplesse de modélisation, permettant l'inclusion des éléments non-standards relevés lors de l'inventaire de la matière dans un processus BIM. Ces nouveaux outils consentent à redonner à l'architecte sa place de créateur. À titre d'exemple la Figure 2 montre la richesse de création que pourrait présenter des éléments de réemploi nonuniformes.
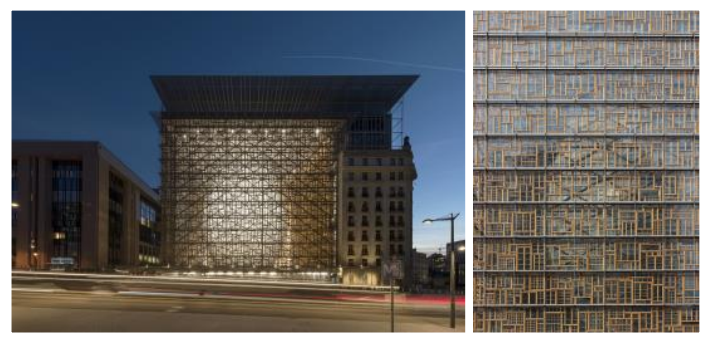

Figure 2. Réemploi de châssis pour le projet European Council par Samyn \& Partners (2016).

\section{Conclusion}

Cet article propose l'architecte comme moteur d'une transition vers les processus BIM et circularité en architecture.

Grâce au réseau d'acteurs comme base commune de travail et aux nouveaux outils de conception qu'apporte l'OPENBIM, l'architecte a la possibilité dans cette révolution BIM6D de faire valoir son expertise créatrice à travers l'unicité du réemploi pour chaque projet. Les nouveaux outils tels que les designers algorithmiques en processus BIM sont un véritable tremplin vers le changement reliant une valeur éthique et une dimension technologique dans un procédé collaboratif.

A la lumière de ces constats, l'intérêt de cette recherche est confirmé mais nécessite une étude de terrain approfondie se basant sur des études de cas pilote BIM6D, sondages auprès d'un panel de bureaux d'architecture et prospection de réemploi.

\section{Bibliographie}

Arayici, Y., Coates, P., Koskela, L.J., Kagioglou, M., Usher, C. and O’Reilly, K., (2011). Technology adoption in the BIM implementation for lean architectural practice. Automation in construction, vol. 20, 2, pp. 189-195.

BAMB2020 , Buildings As Materials Banks 2020. En ligne : http://www.bamb2020.eu

Ben Rajeb, S., Delfosse, V., Boulanger, C., Jeunejean, A., Carre, C., Billen, R. \& Leclercq, P., (2014). Projet de recherche appliquée Spatiodata : Spécification d'un système parti-

\footnotetext{
${ }^{6}$ Nous constatons qu'aujourd'hui encore, le BIM est souvent interprété comme un logiciel et non un processus de travail. Cette confusion a à nouveau été relevée à plusieurs reprises lors des conférences au BIMWORLD à Paris (mars 2018).

${ }^{7}$ BuildingSMART (anciennement IAI ; Internation Alliance for Interoperabiliy) est une organisation internationale visant à améliorer l'échange entre différents logiciels BIM.
} 
cipatif pour la réutilisation de données sporadiques sur le bâtiment, Proceedings du colloque 01'Design.

Delmée, H., (2017). Un vade-mecum pour la conception à partir de matériaux de réemploi, Mémoire de master en architecture non publié, Université catholique de Louvain, Faculté d'ingénierie, d'architecture et d'urbanisme Saint-Luc (LOCI), Bruxelles.

Delmée H., Demol, G., Tordeur R., (2016). Processus d'implémentation de nouvelles techniques constructives, Séminaire critique : Utopie réalisable, Travail d'étude, Université catholique de Louvain, Faculté d'ingénierie, d'architecture et d'urbanisme Saint-Luc (LOCI), Bruxelles.

Directive 2002/91/CE relative à la performance énergétique des bâtiments : directive du Parlement européen et du Conseil du 16 décembre 2002. En ligne :

http://www.controle-peb.be/wp-content/uploads/Directive-Parlement-Europeen.pdf

Directive 2008/98/CE relative aux déchets : directive du Parlement européen et du Conseil du 19 novembre 2008, abrogeant certaines directives. En ligne (consulté le 29 janvier 2018) : http://eur-lex.europa.eu/legal-content/FR/TXT/?uri=celex:32008L0098

Directive 2014/24/UE relative à la passation des marchés publics : directive du Parlement européen et du Conseil du 26 février 2014, abrogeant la directive 2004/18/CE.

En ligne : https://eur-lex.europa.eu/legal-content/FR/TXT/?uri=celex\%3A32014L0024

Europa (2018). En ligne http://www.europarl.europa.eu, consulté le 21 janvier 2018.

Gobbo, E., (2015). Déchets de construction, matières à conception, Analyse des stocks et flux de matières dans le cadre des opérations de rénovation énergétique en Région de Bruxelles-Capitale, Thèse de doctorat, Faculté d'architecture, d'ingénierie architecturale, d'urbanisme (LOCI), Louvain-la- Neuve, Belgique.

Guide pratique du réemploi réutilisation des matériaux de construction (2013), dans le cadre de l'Alliance Emploi - Environnement. En ligne sur le site de l'Université de Liège, Unité CIFFUL :

http://www.cifful.ulg.ac.be/images/stories/Guide_reemploi_materiaux_lecture_2013.pdf

Lame, G., \& Leroy, Y., (2015). Écoconception des bâtiments : pratiques actuelles et freins à l'utilisation des outils d'écoconception. Une étude en France dans le cadre du $14 \mathrm{e}$ Colloque National AIP-Priméca, La Plagne.

Lebègue E. et Segura J-A. C., (2015). Conduire un projet de construction à l'aide du BIM, Ed. Eyrolles.

Marin P., et Segura J.-A. C., (2014). Outils, méthodes et acteurs : analyse des limites à l'utilisation de la maquette numérique, SCAN14 - Actes du Séminaire de Conception Architecturale Numérique.

Picon, A., (2016). Architecture et culture numérique, évolution ou révolution. Conférence à LOCI, Université Catholique de Louvain, novembre 2016. 